\title{
Miranda
}

Revue pluridisciplinaire du monde anglophone /

Multidisciplinary peer-reviewed journal on the English-

speaking world

\section{$22 \mid 2021$}

Unheard Possibilities: Reappraising Classical Film Music Scoring and Analysis

\section{Philippe Goudard et Nathalie Vienne-Guerrin (eds.), Figures du clown, sur scène, en piste et à l'écran}

\section{Raphaëlle Costa de Beauregard}

\section{(2) OpenEdition}

Journals

Édition électronique

URL : http://journals.openedition.org/miranda/38081

DOI : 10.4000/miranda.38081

ISSN : 2108-6559

\section{Éditeur}

Université Toulouse - Jean Jaurès

Référence électronique

Raphaëlle Costa de Beauregard, «Philippe Goudard et Nathalie Vienne-Guerrin (eds.), Figures du clown, sur scène, en piste et à l'écran », Miranda [En ligne], 22 | 2021, mis en ligne le 17 mars 2021, consulté le 27 avril 2021. URL : http://journals.openedition.org/miranda/38081 ; DOI : https://doi.org/10.4000/ miranda.38081

Ce document a été généré automatiquement le 27 avril 2021.

\section{c) $(9)$}

Miranda is licensed under a Creative Commons Attribution-NonCommercial-NoDerivatives 4.0 International License. 


\section{Philippe Goudard et Nathalie Vienne-Guerrin (eds.), Figures du clown, sur scène, en piste et à l'écran}

\section{Raphaëlle Costa de Beauregard}

\section{RÉFÉRENCE}

Philippe Goudard et Nathalie Vienne-Guerrin (eds.), Figures du clown, sur scène, en piste et à l'écran (Montpellier : Presses Universitaires de la Méditerranée, 2020), 474 p., 83 ill. noir et blanc et couleurs, ISBN 978-2-36781-332-5

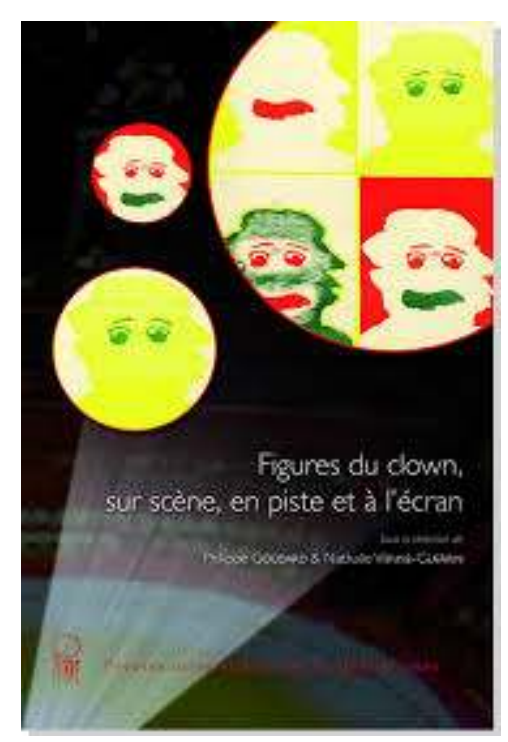

Dans l'introduction, Philippe Goudard et Nathalie Vienne-Guerrin proposent une analyse lexicographique, scénique et artistique du mot clown : infixable et insaisissable sont les deux traits caractéristiques de la figure. Le commentaire d'une importante 
bibliographie et une approche pluridisciplinaire ont motivé le choix des textes et leur organisation en six parties, dans le cadre d'un programme de recherche Cirque : histoire, imaginaires, pratiques, Université Paul Valéry, Montpellier 3 et d'un colloque Figures $d u$ clown, sur scène, en piste et à l'écran.

\section{Le clown élisabéthain}

2 Le clown élisabéthain est à la fois une figure fondatrice d'un théâtre dont la modernité se veut universelle et un personnage fascinant dont la fonction est centrale car il doit toujours communiquer avec le public. Le clown --alias le fou - est, pour cette raison, la source d'effets comiques, entre travestissement et satire, permettant la libération de la parole par le rire. Les considérables lacunes documentaires, comme le rappelle Yan Brailowsky dans son article «'Where's my knave, my fool ?' à la recherche du clown élisabéthain ", ainsi que le thème même de la disparition de clowns dans ce théâtre qui a pourtant connu des acteurs célèbres tels que Armin et Tarlton, sont autant d'obstacles à poursuivre ce domaine de recherche. Par ailleurs, avec les adaptations de Shakespeare au XXe siècle apparaît un phénomène remarquable, souligne Yan Brailowsky, celui des femmes dans le rôle des clowns. D'un point de vue lexicographique, Nathalie Vienne-Guerrin, avec «Le 'clown' et ses clones dans le théâtre shakespearien : approche lexicale », montre que le mot clown couvre un vaste champ sémantique où se dessinent d'une part, l'opposition public éduqué/public populaire, et, d'autre part, une utilisation paradoxale hésitant entre éloge et injure. À sa suite, des mises en scène récentes du clown sont étudiées : à Avignon, Florence March avec «Le clown shakespearien sur la scène contemporaine: l'héritage élisabéthain revisité au Festival d'Avignon » et à Stratford, Nathalie Vienne-Guerrin " La femme (du) Clown: Audrey dans Comme il vous plaira ». Cette dernière offre pour conclure cette partie du volume une définition des « clowns élisabéthains entre corps et esprit ».

\section{Femmes clowns : fantasme, fiction, réalité}

L'étude " Écrire les femmes de cirque entre pratiques culturelles et discours raciaux : la clowness Lulu de Félicien Champsaur» que consacre Filippos Katsanos au roman clownesque de Félicien Champsaur Lulu (1901), fournit une introduction éclairante à cette deuxième partie du volume consacrée aux femmes clowns - dont on a déjà vu la présence sur la scène shakespearienne moderne. Lulu dans ce roman est une acrobate clownesque aussi talentueuse que ses homologues masculins; le personnage aurait une origine double: un acrobate britannique qui se produisait à Londres depuis 1870 sous ce nom, Sam Farini, et une contorsionniste, clownesse et danseuse parisienne Cha-UKao, dont Toulouse-Lautrec nous a laissé le portrait. Le roman abandonne toute tradition androgyne et souligne la féminité de Lulu, car le personnage bénéficie d'une extrême érotisation. Pour le lecteur bourgeois français de la Belle Époque sous la IIIe république, Lulu est l'emblème des nouvelles vedettes féminines des spectacles de cabaret et de music-hall telles que Louise Weber. Cependant Lulu est surtout une femme indépendante qui incarne à la fois Vénus et Diane pour son public masculin. On reconnaît en elle un stéréotype qui fit fortune au cinéma, celui de la femme fatale qui transforme en 'pourceaux' les hommes qui se laissent fasciner par elle. L'article 
s'appuie sur de nombreuses sources de la presse de l'époque qui permettent une approche sociologique de ce personnage de roman.

4 Dans son article «Clowns, figures instables: photographies de Cindy Sherman et Roni Horn ", Franck Leblanc s'intéresse au travail sur la figure du clown, à la fois comme visage et comme corps en mouvement. À partir de la série photographique de Cindy Sherman, Clowns (2003-2004), l'auteur montre comment cette double dénotation du mot clown est la source de l'inspiration de l'artiste. Le maquillage et le corps burlesque créent un personnage comique, mais c'est surtout le travail photographique de Roni Horn qui retient l'attention par son souci paradoxal de fixer le mouvement. Le temps long de l'exposition de la pellicule laisse « un sillage argentique sur son passage » (142), dans le but d'exprimer visuellement le concept d'instabilité qui serait le propre du clown.

5 Une approche de l'expressivité du clown, et en particulier de la femme clown, comme performance est proposée par Béatrice Picon-Vallin par une étude des clowns du Théâtre du Soleil d'Ariane Mnouchkine «Les femmes clowns au Théâtre du Soleil ». En se voulant clown, la femme se marginalise. Mais dans la recherche de cette troupe, le travail de chaque artiste étant de formuler un personnage unique, la femme clown y trouve une occasion de se libérer des préjugés en accédant au statut d'artiste performatrice. Ce texte est à lire en parallèle avec celui de Guy Freixe sur Jacques Lecoq. Le même sujet est abordé par Amélie Chabrier qui, avec «Nez à nue : quand le clown rencontre la sensualité ", étudie le spectacle de Sabrina Maillé et son travail sur la sensualité du corps clownesque, tandis que Sandy Sun, "Zouc, clowne de théâtre » évoque l'artiste Zouc, qui se produisit dans les années 1970, et dont l'influence se retrouve chez Muriel Robin et Laetitia Rosch. Un grand nombre de références à des sites internet permettent au lecteur de trouver des images du travail de ces artistes.

\section{Les clowns au tournant du XXe siècle}

6 Dans cette troisième partie, si brève qu'elle soit, l'essentiel du clown moderne, et la création du clown de cinéma, sont présentés. Krizia Bonaudo, « Les clowns dans l'avantgarde théâtrale française entre 1990 et 1924 ", rappelle comment au début du siècle, la figure du clown a éveillé l'intérêt des producteurs de spectacle pour qui il y avait là un modèle artistique et non une simple attraction foraine. C'est l'entrée sur scène, numéro dont les clowns se sont fait une spécialité, qui leur vaut cette nouvelle appréciation de leurs performances. La tradition y voyait un moyen de divertir le public par des culbutes et autres chutes comiques pendant un interlude entre deux spectacles, qu'ils soient spectacles équestres, ou animaux sauvages et leur dompteur, ou trapézistes. Pour les avant-gardes théâtrales du début du XXe siècle, et des auteurs tels que Guillaume Apollinaire ou Tristan Tzara, le cirque, mais surtout le clown, incarnent la liberté d'expression. Krizia Bonaudo cite à titre d'exemple Parade (1917) de Jean Cocteau comme modèle de cette mouvance où il s'agit d'exprimer cette liberté en tirant parti de l'expressivité du corps clownesque au cours de chutes simulées, d'acrobaties, voire de tapage. Or cet éveil d'intérêt pour le spectacle clownesque est contemporain de la naissance du cinéma, art du mouvement s'il en est. Le clown est rapidement devenu une figure de l'essence du cinéma, la prise de vue fixe restreinte à la scène tirant parti de la piste même du cirque, et de la figure centrale du clown. Dans l'article «Clowns au cinéma, clowns de cinéma (1895-1926) », François Amy de la Bretèque rappelle que c'est 
le cinéma français qui a créé ce personnage si particulier, le clown de cinéma. Lumière, mais surtout Méliès, ont fait du clown une figure cinématographique vouée à une nombreuse descendance. Christian Rolot et Francis Ramirez évoquent ensuite dans ce même article ce cinéma américain qui y trouva rapidement une source d'inspiration avec des figures de clowns solitaires qui seraient des 'augustes', créés par Chaplin, Langdon ou encore Arbuckle. L'extraordinaire capacité de Chaplin à mêler le rire et les larmes fit de lui bien plus qu'un simple clown. Il veilla cependant « soigneusement à ce que le vagabond pathétique ne l'emportât jamais sur l'auguste espiègle qui, par ses facéties, savait si bien retendre la peau des mélodrames » (223). Langdon créa la figure du petit clown malicieux mais sans méchanceté, et Arbuckle, dit 'Fatty', était un excellent cascadeur qui tirait parti de son poids redoutable. Le clown de cinéma donna aussi naissance à des duos tels que Laurel et Hardy, où le clown blanc et l'auguste se donnaient la réplique, ou plus tard Jerry Lewis et Dean Martin. Buster Keaton ne fit jamais référence au cirque, contrairement à Chaplin, (voir l'article de Francis Ramirez qui est consacré à ce grand clown de cinéma dans la $4^{\mathrm{e}}$ partie ci-dessous).

\section{Figures de clowns}

7 À lire les difficultés d'un artiste comme Medrano de se faire connaître, ou Rafael alias Chocolat selon Gérard Noiriel, on mesure à quel point le passé vécu nous échappe, ce dont témoignait déjà Yan Brailowsky. Cependant ce qui intéresse Marie-Eve Thérenty, "Medrano (Boum-Boum) : construction d'une figure médiatique", c'est, a contrario, l'utilisation de la presse dont Medrano s'est emparé pour s'inscrire dans l'histoire de la société, au point, écrit l'auteur de faire oublier ses talents d'artiste. Gérard Noiriel étudie également Chocolat, dont Toulouse-Lautrec nous laissé un portrait vantant les talents d'artiste, du point de vue sociologique, montrant son statut de pur divertissement dans la société de son temps peu capable d'apprécier ses talents exceptionnels qui lui valurent d'être célèbre de son temps justement. Quant aux Fratellini, troisième figure de clowns de cette $4^{\mathrm{e}}$ partie, c'est leur généalogie autant que leur contribution à la modernité de la figure dans un exemple particulier, un film de René Henrion sous leur direction, Rêves de clown (1924), qui intéresse ici Philippe Goudard: "Les Fratellini, clowns et figures de modernité ». L'approche biographique qui se prête à un commentaire sociologique dans ces pages témoigne de l'écart entre une mémoire collective où s'affichent quelques noms célèbres et la nature éphémère des spectacles clownesques, à l'exemple de Grock, étudié par Olivier M. Meyer dans le quatrième article de cette partie de l'ouvrage «Disparu mais présent: où est passé Grock?».

8 Une deuxième série d'articles consacrés aux figures de clowns se détourne de l'analyse sociologique à proprement parler pour interroger les caractéristiques artistiques de certaines figures de clown qui les ont inscrits dans la mémoire collective. C'est ainsi qu'est republié un article plus ancien de Francis Ramirez consacré à Buster Keaton intitulé «Comique et beauté : la ligne Keaton» (271-285), texte qui sert en quelque sorte de pivot au cœur de ce volume collectif. Ce qui constituerait le clown en figure de la mémoire collective serait le contact entre l'artiste et son public, par le regard caméra par exemple puisqu'il s'agit, déjà, de cinéma, tout en sachant s'élever jusqu'à la poésie, et, partant, jusqu'au mythe. Déjà dans ce volume, on a pu lire une réécriture du mythe 
de l'éternel féminin par les femmes clowns. Il est donc nécessaire d'étudier le jeu clownesque en lui-même au même titre qu'en littérature on étudie l'écriture poétique.

Le travail de Jacques Lecoq, et avant lui de Copeau, et après lui, de Mnouchkine, fait l'objet d'une étude de fond par Guy Freixe: «Jacques Lecoq et l'enseignement du clown ». Dès Copeau et la période 1914-18, un renouveau du clown de cirque se fait jour qui devient clown de théâtre au sens d'une expressivité des sentiments d'un sujet particulier. Or cette expressivité reposera désormais sur l'improvisation de la gestuelle de l'artiste clown et non, comme au théâtre, de la transcription d'un texte. Il s'agit en effet d'une poétique du geste, c'est-à-dire du mouvement et donc du rythme. Le concept de l'acteur créant son propre clown, que développe Jacques Lecoq, dont l'emblème est le seul nez rouge (290), est bien celui que développe Mnouchkine dont il a déjà été question. Ce texte éclaire donc le thème de la modernité du clown par l'étude détaillée de cette nouvelle poétique, ce qui permet au lecteur de mieux appréhender les textes qui suivent consacrés aux spectacles de troupes de clowns tchèques et russes.

Les quatre articles qui terminent cette partie consacrée aux figures de clown présentent deux écoles de clown d'une très grande richesse dans la liberté de l'improvisation. La première école de clowns est tchèque et Ctibor Turba lui consacre à une présentation détaillée avec l'exemple du Cirque Alfred : «Les clowneries du Cirque Alfred». C'est le metteur en scène et créateur du cirque qui nous présente l'art de sa troupe dans son spectacle baptisé Klaunerie. Trois tableaux sont décrits - le quatrième est donné dans le second texte sur le sujet- tout d'abord l'entrée en scène de musiciens et les pantomimes jouant leur expectative, leur performance et la suppression de leurs accessoires par deux policiers. La disparition des accessoires met en évidence le sujet du spectacle de clown: art de l'improvisation, de l'expression de la gestuelle, et la liberté de l'imagination pour compenser la disparition des objets : le banc supprimé est remplacé par un clown servant de siège aux autres, les petites cymbales confisquées sont remplacées par un claquement de mains, et ainsi de suite. Le second tableau joue avec le cercle de lumière de la piste comme une matière abstraite avec laquelle les clowns jouent : elle rétrécit, s'amplifie, et ils se déplacent, puis elle devient un objet qu'ils manipulent. Le troisième tableau intitulé 'Le Papier' décline là encore toutes les possibilités de jeu offertes par cette matière. Du petit bout de papier qu'il faut ramasser au rouleau de papier, les clowns rivalisent pour s'en emparer ou le modifier. Katerina Vlckova avec « Notes sur la création de 'Klauneire' du Cirque Alfred» reprend ensuite le contexte historique tchécoslovaque et les périodes de censures interdisant les spectacles de clowns. Elle complète la description de Klaunerie par un dernier tableau encore plus extraordinaire, jouant sur une autre matière abstraite, le son. Un son d'origine inconnue est l'objet d'une poursuite et d'une recherche par les clowns dans plusieurs sketches, puis, croyant l'avoir trouvé et attrapé, ils finissent par l'enterrer dans un pot de fleurs. Le son joue alors sa propre marche funèbre.

11 Les deux articles suivants présentent le même modèle de performance fondé sur l'improvisation, l'imagination et la poésie, celui du clown russe et sa place dans la culture russe et l'on note que le contexte est tout à fait différent. Béatrice Picon-Vallin rend compte de l'œuvre et de la carrière de Viatcheslav Polounine, clown qui est un artiste reconnu et valorisé en URSS avec «Slava Polounine: le clown et la théatralisation de la vie». Le sujet de son spectacle Slava's Snowshow dont elle rend compte est le rêve de liberté incarné par moult bulles, balles et ballons de toutes tailles avec lesquels jonglent les clowns avant qu'elles n'envahissent les spectateurs qui 
cherchent sans succès à s'en emparer. Polounine cherche à tirer le maximum de poésie de ce désordre, l'espace du spectacle devenant un espace d'apparitions. L'environnement tant par l'éclairage que par le son est constamment mouvant. Polounine aspire même à une théatralisation de la vie, et dit qu'il faut « envisager la vie comme un art " (343). Le théoricien russe contemporain Nikolai Evreinov, également praticien et metteur en scène, a publié des ouvrages qui développent toutes ces idées, Le Théâtre en tant que tel, ou encore Le Théâtre pour soi. Béatrice Picon-Vallin fait ensuite une présentation générale de l'école russe des clowns au XXe siècle «L'école ruse des clowns ». Des clowns acrobates et satiristes voient le jour avec l'avant-garde théâtrale de Maïakovski et Meyerhold jusqu'à Polounine dont il vient d'être question. On remarque que ces quatre textes obéissent à une règle simple, d'abord donner un exemple étudié en détail, puis le situer dans un contexte historique plus général, et non l'inverse comme on pourrait s'y attendre.

\section{Tragique : l'autre côté du clown}

12 Cette cinquième partie examine l'autre face du clown à partir de figures de clowns qui se caractérisent par la possibilité d'un tel retournement. Philippe Goudard dans " Rigoletto clown tragique », nous rappelle comment le duo clown et figure du pouvoir permet de mettre en scène cette ambivalence de la figure entre merveilleux et horreur, sublime et grotesque. En 2014 l'opéra de Verdi Rigoletto d'après la pièce de Victor Hugo Le roi s'amuse a été mis en scène avec un cirque et non un palais. On n'en saura pas plus sur cet événement, hélas, mais la généalogie du clown qui est rappelée en détail peut expliquer comment ce choix a été préféré aux mises en scène traditionnelles de cet opéra. L'histoire de la figure clownesque commence avec le vice médiéval, auquel succède le fou élisabéthain. Au $19^{\mathrm{e}}$ siècle le duo blanc/auguste visualise les deux faces du clown, le premier étant un acrobate aristocratique et le second un misérable au nez rouge de froid et de boisson. La pièce de Hugo et l'opéra de Verdi furent censurés pour leur caractère subversif, comme si le fou ne servait plus de faire valoir mais passait sur le devant de la scène comme un personnage tragique, perdant son statut de clown blanc à celui de clown auguste par la cruauté de son destin (Rigoletto tue sa propre fille par erreur). Le choix d'une piste de cirque pour l'opéra de Verdi en 2014 semble donc symboliser l'enfermement du personnage dans un destin tragique, ce qui est le thème central du film de Sjöström, He who Gets Slapped (1924) étudié par Marion PoirsonDechonne «Larmes de clown ». Dans cette adaptation à l'écran d'une pièce de théâtre russe mise en scène à Paris par les Pitoëff en 1921, le personnage est prisonnier de ce cercle circassien où il décide pourtant librement de s'enfermer dans l'anonymat à la suite d'une cruelle humiliation en public. Ce cercle maudit est un motif important du film, par sa répétition assurant plusieurs fondus enchainés et comme raccord entre des séquences. On retrouve ce motif dans les cadrages souvent de face du visage maquillé du clown blanc, tandis que nous savons qu'il est en réalité un auguste torturé par la cruauté de son destin. Dans ce cas comme dans le précédent, c'est la Mort que, tout clown qu'ils sont, les deux personnages ne peuvent déjouer. Lorsque le clown devient maléfique dans une société gouvernée par les Puritains, sujet de l'article de Florent Christol, «Le clown maléfique au cinéma et dans la culture américaine : généalogie », il inspire la terreur et devient étranger à sa fonction première de faire rire. L'auteur reprend la généalogie de la figure clownesque où l'avait laissée Philippe Goudard pour évoquer ce clown terrifiant qui relève du gothique américain. La diabolisation du clown 
en Amérique du Nord, entraînant une véritable phobie du cirque et ses clowns, baptisée 'coultophobie', mène tout naturellement à substituer au spectacle de la performance par un artiste celui de l'exhibition de monstres. C'est le 'freak show', qui a pour fonction d'ériger un rempart entre le spectacle et le spectateur. On voit d'ailleurs le clown blanc se grimer en 'black face' supposé faire rire ce spectateur avide de divertissement.

\section{Rire}

Dans l'article du sémioticien Paul Bouissac «Quand et pourquoi le spectateur rit-il ?», l'auteur explique que c'est en dépassant la description tautologique : on rit par ce que c'est comique et/ou c'est comique parce ce que l'on rit, que l'on pourra répondre à cette question. Après une étude de cas, celle de la performance du clown de cirque Rob Torres qui provoque différents types de rires, l'auteur propose plusieurs approches. L'approche des neurosciences tout d'abord: «le rire est un phénomène physiologique qui dépend de réseaux neurologiques qui [...] peut être décrit comme une décharge d'énergie nerveuse » (406), complétée par une approche situationnelle qui souligne que la soudaineté de l'événement clownesque est cruciale. D'un point de vue différent, l'approche causale est proposée pour étudier ce comportement chaotique: ce paradigme décline ainsi la cause sensorielle, telle que le chatouillement, la cause cognitive telle que la révélation de l'inattendu, car celui-ci est étranger à notre anticipation, ou encore la révélation de l'inacceptable, et une confrontation avec nos inhibitions culturelles. Une approche psychologique montre enfin que ce comportement est tantôt la cause d'un sentiment d'euphorie, tantôt son contraire la dysphorie. En somme, le rire serait un ricochet causal accidentel. Reprenant le même sujet, le psychanalyste Eric Smadja se fait l'écho de ce premier texte, avec son article " Le rire, un exemple de la complexité humaine: une approche pluri et interdisciplinaire». Il pense que tout comportement humain, mimiques faciales, et autres techniques corporelles «doivent faire l'objet d'une approche globale, pluridisciplinaire, comportant les dimensions biologique, psychologique et socioculturelle » (413-14). Étudiant d'abord le comportement du rire, il propose une typologie allant du rire silencieux jusqu'au fou rire en passant par la vocalisation interne, le rire vocalisé, et le rire explosif. Dans tous ces cas, il s'agit chez l'individu du développement d'un comportement instinctif dès les premiers instants de sa vie, qui est rendu possible par une fabrication d'ordre psychique, cognitif et psychoaffectif. On voit apparaître tout au long de ce développement accompagnant la croissance de l'individu, un stimulus cérébral spécifique, le risible, identifié grâce au cortex frontal et ses lobes préfrontaux comme l'ensemble 'risible-rire' et accompagné d'une compétence comportementale grâce à des substances comme l'adrénaline et la dopamine. En outre, le rire comme expérience corporelle est lui-même source de plaisir par le relâchement musculaire, la stimulation du système nerveux et la baisse du rythme cardiaque, entre autres effets. En parallèle, poursuit Eric Smadja, le 'risible-rire' est un système de communication, car il s'inscrit dans un cadre spatio-temporel, intègre émetteur (du risible) et récepteurs (rieurs), tout en véhiculant des thèmes et des techniques risiblerires, à partir d'objets transformés : humains mécanisés, animaux humanisés etc... Ces pratiques ont des fonctions psychiques, telles que satisfaire les pulsions sadiques de l'individu, et sociales, telles que satisfaire ses pulsions exhibitionnistes, ainsi que des fonctions intellectuelles par la transgression, l'exclusion ou l'intégration. Et enfin, il 
s'agit d'une défense contre l'angoisse de la mort par une brève mais sonore métaphore facio-vocale symbolisant l'éphémère de la vie.

Après un rappel des différentes recherches sur le rire et une synthèse des deux articles précédents, Philippe Goudard, créateur, producteur et interprète d'œuvres de cirque, nous fait part de son expérience personnelle avec « Faire rire : le clown à l'œuvre ». Il s'intéresse au surgissement du clown en nous, lequel, bien loin de nous protéger sous le masque d'un personnage, nous expose à l'expérience de la nullité (439). Tout d'abord les formes qui contribuent à l'échange avec le spectateur, les types culturels, sont inventoriées comme autant de choix possibles. Puis une esquisse d'une dramaturgie clownesque est proposée pour répondre à l'essence clownesque du désordre ordonné. Si la composition clownesque passe de l'improvisation à une forme d'écriture, il est surtout essentiel de dire qu'une entrée clownesque ne connait pas de préambule, car dès qu'il apparaît il est déjà en prise avec une situation ou un objet. Le contact avec le public est direct et interactif, le rythme des actions capital, avec une importance particulière des variations soudaines. D'un point de vue dramatique, comme l'acrobate, le clown se place dans des situations réputées insolubles, et lorsqu'une solution apparait, elle déclenche une cascade de conséquences plus catastrophiques encore. Les clowns jouent en effet avec le déséquilibre à la fois gestuel et comportemental. Il s'agit donc d'organiser le désordre.

Les clowns "sont les génies d'un monde à l'envers où le retournement de l'existence fait un pied de nez à l'angoisse de la mort » écrit Philippe Goudard à titre de conclusion (454). Ne serait-ce que pour cette raison, les articles référencés ci-dessus constituent dans leur ensemble un remarquable volume à lire et décrypter dans le détail avec délice.

\section{INDEX}

Keywords : clown, circus, theatre, cinema, Elizabethan, female-clowns, modernity, tragedy, laughter

Mots-clés : clown, cirque, théâtre, cinéma, élisabéthain, femmes-clown, modernité, tragédie, rire

\section{AUTEURS}

\section{RAPHAËLLE COSTA DE BEAUREGARD}

Professeur Emérite

Université de Toulouse 2-Jean Jaurès

costa_de_beauraph@orange.fr 